\title{
Evolutionary algorithm based 9DOF sensor board calibration
}

\author{
Peter Sarcevic*, Szilveszter Pletl**, Zoltan Kincses ${ }^{* * *}$ \\ ${ }^{*}$ University of Szeged, Department of Informatics, Szeged, Hungary \\ ${ }^{* *}$ University of Szeged, Department of Informatics, Szeged, Hungary \\ ** Subotica Tech, Department of Automation, Subotica, Serbia \\ *** University of Szeged, Department of Informatics, Szeged, Hungary \\ sarcevic@inf.u-szeged.hu, pletl@inf.u-szeged.hu, kincsesz@inf.u-szeged.hu
}

\begin{abstract}
Accelerometers, gyroscopes and magnetometers can be used in a large variety of applications, and their calibration is a very actual problem due to high error rates, especially when errors are integrated in time. Systematic errors from the measurement values can be removed by sensor calibration, thus the applicability of the sensor can be increased. In this paper, a new evolutionary algorithmbased, quick and easy-to-use calibration method is presented. The algorithm has been developed and tested with real measurement data of the above-mentioned sensors. During this work, measurement data have been collected with 9 degree of freedom (9DOF) sensor boards, which are built up of three-axis accelerometer, gyroscope and magnetometer. For accelerometer and magnetometer calibration, bias values, scale factors and non-orthogonality corrections have been calculated, while for the gyroscopes only offsets have been determined.
\end{abstract}

Keywords-sensor calibration, accelerometer, gyroscope, magnetometer, evolutionary algorithm

\section{INTRODUCTION}

Systematic errors of sensors lead to differences between measured and real values, and these errors affect every new measurement. These errors can be calculated during sensor calibration and they can be compensated later during the real-time work.

The 9 degree of freedom (9DOF) sensor boards are built up of three digital three-axis sensors: accelerometer, gyroscope and magnetometer. These sensors are nowadays used in a large number of applications, and their calibration is subject of intensive research.

Low-cost MEMS (micro-electro-mechanical system) three-axis accelerometers measure acceleration in three dimensions. Accelerometers can be calibrated using the fact that the vector magnitude should be $1 \mathrm{~g}$ when the sensor is not in movement as written in [1]. As a result of the calibration process, scale factors, bias values and axis misalignments can be calculated. Bias in the accelerometer output will cause a shift in the measured acceleration vector from its real direction. Scale factors determine the sensor's sensitivity to the intended acceleration at each axis. In the case of an ideal three axis sensor: the sensor is linear, all axes are orthogonal to each other, all axes are linearly independent, the sensitivity is the same for all independent directions and the bias vector is zero. But real sensors are not ideal.

Three-axis gyroscopes measure angular velocity around three axes. Bias errors have a big impact on overall performance, and they can be removed by stationary measurements. Scale factors and misalignment errors of gyroscopes cannot be determined with these measurements. To define them, complex and precise measurement systems are required.

Three-axis magnetometers provide information about the direction and magnitude of the magnetic field. Regarding magnetometers, the concept is the same as in the case of accelerometers, but the magnitude is not known, and movement does not affect it.

\section{SENSOR CALIBRATION METHODS}

\section{A. Accelerometer}

Calibration of accelerometers and magnetometers can be reduced to $3 \mathrm{D}$-ellipsoid fitting problems. In [2] a noniterative algorithm has been proposed which focuses on minimal execution time and low memory consumption. The method works in two steps: first, the center of the ellipsoid is estimated, and then the scale factors are computed. The aim of both steps is to form and solve a system of linear equations with the same number of equations and unknown variables using the least-square method.

In [3] a calibration method for scale and cross factors and a dynamic filtering solution for the bias have been proposed.

Using different sensor models, calibration methods and parameter numbers, an autocalibration method has been described in [1].

\section{B. Gyroscope}

The biggest error sources of the angular rate sensors are the offsets. For the calibration of sensitivity and misalignment factors, laboratory equipment is needed.

In [4], the calibration of an accelerometer is done online. The uncalibrated accelerometer delivers attitude errors, and to correct these errors, a multi-model error state Kalman filter has been proposed for determining the gyroscope`s offset.

A miniature magnetic and inertial measurement unit, containing an accelerometer, a gyroscope and a magnetometer, has been developed in [5]. This sensor system was calibrated and aligned with simple but effective procedures. During gyroscope calibration, the bias has been determined first. The bias vector was estimated as the mean value of the sensor data during the measurement period, where the system was kept standstill. 
The other nine parameters were determined by making three other measurements for each parameter triplets. Each measurement has been done by rotating the sensors around the sensitivity axes with a constant angular rate. The measured data were then corrected by the bias values, integrated, and transformed into the angle estimate. The parameters have been determined using the estimated and true rotation angles.

In [6] the calibration of a low-cost inertial measurement unit has been studied. For gyroscope calibration, only a simple rotating table was required. The proposed method utilizes the fact that ideally, the norm of the measured output of the accelerometer and gyroscope is equal to the magnitude of the applied force and rotational velocity. This fact with the sensor models was used to construct a cost function which has been minimized with respect to the unknown model parameters.

The Gauss-Newton iterative nonlinear regression method was used in [7] to calculate calibration parameters.

\section{Magnetometer}

The full magnetometer calibration process involves the determination of scale factors, bias values and nonorthogonality corrections.

In [8] two real-time algorithms for magnetometer calibration have been proposed based on the extended Kalman filter and Unscented filter. The simulations and experimental results showed that the Unscented filter is significantly better than the extended Kalman filter.

A maximum likelihood estimator is formulated in [9] to iteratively find the optimal calibration parameters that best fit to the sensor readings. The algorithm has been tested with 60000 points which have been obtained by using a 2axis and a single-axis magnetometer. The results showed that the calibrated readings are near to the unit circle.

In [10] a non-linear, two-step estimation algorithm for calibrating solid-state strapdown magnetometers has been presented. The estimated errors were used to calibrate the magnetometer.

A non-magnetic rotation platform and the differential evolution algorithm have been used in [11] for three-axis magnetometer calibration. The performance of this method was analyzed using simulation and real experiments, and compared with the unscented Kalman filter, recursive least squares and genetic algorithm. The results showed that the differential genetic algorithm has the least calibration error and the best robustness. In addition to these advantages, the algorithm is not sensitive to initial parameters, which is an advantage compared to other iteration algorithms.

In [12], three different algorithms for three-axis accelerometer calibration have been implemented, tested and compared with each other. The results showed that the performance of symmetrical calibration is better than that of orthogonal and random calibration.

In [13] an adaptive least squares estimator has been used for the ellipsoid fitting problem. In this method the Canadian Geomagnetic Reference Field has been used for the sphere's radius since the experiment has been performed in Calgary (Canada).

A particle swarm optimization (PSO) algorithm-based error calibration method is presented in [14], where the calculation of bias and scale factor errors has been performed. The errors have been significantly reduced, and the simulations showed fast convergence speed and high accuracy.

\section{THE USED HARDWARE AND SOFTWARE COMPONENTS}

The future goal of the research project is to detect medical emergencies. To perform this task a flexible and easy-to-program wireless sensor mote is required, as on the one hand, the user should not be disturbed in free movement, and on the other hand, the possibility of fast prototype development is needed. Based on these considerations the IRIS wireless sensor mote was selected, which is a widely used platform for the implementation of different Wireless Sensor Networks (WSNs).

The IRIS mote is built up of four main parts, an ATmel Atmega 1281L 8-bit microcontroller, an RF231 IEEE 802.15.4 compatible radio transceiver, a 512Kbyte SPI Flash, and a 51-pin expansion connector. The maximal data throughput of the radio transceiver is $250 \mathrm{kbps}$, and it has outdoor range over $300 \mathrm{~m}$. Several different sensor boards can be connected to the IRIS mote via the 51-pin expansion connector, i.e. an MTS300/320, MTS400/420 or an MDA100 prototyping board.

To perform the measurements, a 9DOF sensor board was connected to the IRIS mote using the MDA100 prototyping board. The 9DOF sensor board is built up of an ADXL345 accelerometer, an ITG3200 gyroscope and an HMC5883 magnetometer. The IRIS mote and the connected sensor board can be seen on Fig. 1.

In the field of WSNs, TinyOS is the most widely used event-driven operating system. To configure the sensors and read the measured data via $\mathrm{I}^{2} \mathrm{C}$ interface, a TinyOScompatible sensor board driver was implemented using nesC language. In addition, a TinyOS-compatible application was also created, which can run on the IRIS wireless sensor board. The main tasks of this application are to cyclically read the measured data using the sensor board driver, and to send them via the wireless radio. On the receiver side, a mote connected to a PC via MIB520 programming board was applied. On this mote, a so-called BaseStation application was implemented. The main task of this application is to send the received data via the radio to the PC using a virtual serial port.

On the PC, the received data were saved into a MySQL database, because using the appropriate parameterization it is flexible, robust and fast enough for this application.

The highest sampling rate of the three-axis gyroscope

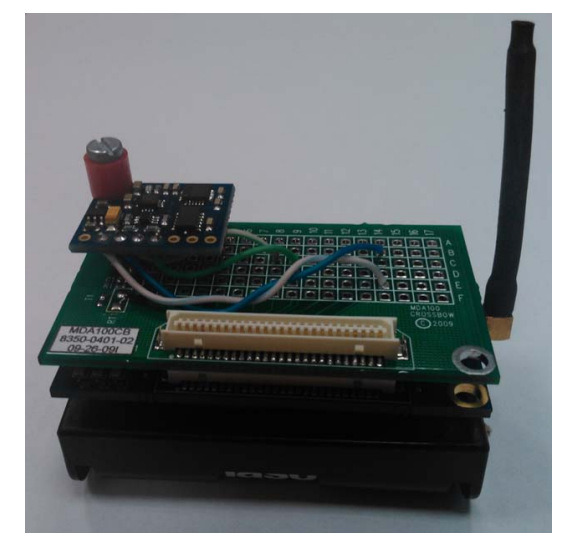

Figure 1. IRIS mote with the attached sensor board 
located on the 9DOF sensor board is $8 \mathrm{kHz}$, the three-axis accelerometer can perform at $3200 \mathrm{~Hz}$, while the three-axis magnetometer can be sampled only with $160 \mathrm{~Hz}$. Since the readout of the data should be in the same time instants, the readout frequency was adjusted to the slowest sensor. Thus, the applicable sampling rate became $125 \mathrm{~Hz}$, which will be fast enough to detect hand gestures and different motion sequences. The size of one data package generated by the three sensors is 18 bytes. This implies that about $17 \mathrm{~kb}$ data should be transmitted per second. Since the data bandwidth of the radio transceiver of the IRIS mote is $250 \mathrm{kbps}$, it is more than enough for the transmission of the generated data.

On the receiver side the maximal data bandwidth of the BaseStation application is 115200 bits, so several wireless sensor motes can be used in parallel to send the measured data to the receiver which then store them to the MySQL database.

\section{ALGORITHM DESCRIPTION}

In the first step measurements are made and the data are saved into a MySQL database. For the realization of a successful calibration, sufficient samples are needed in different orientations of the sensor board. So in this step three IRIS motes with sensor boards using the same TinyOS driver and application were set up, and measurement data were recorded in 80 different stationary orientations with every mote. In every orientation 200 samples were saved. In addition, 5000 magnetometer measurements were performed with each sensor board while the sensors were rotated, to record values on the entire surface, not only in certain points.

In the next step, parameter selection and fitness function construction is performed. If the application does not need very precise values, smaller number of parameters can be used. The used fitness function should be constructed depending on the used sensor model, and on what kinds of errors should be calibrated Thermal calibration has not been realized, because in the future project the sensors will work at room temperature at which the calibration has been done.

The parameters are double type values, and they form the phenotype for optimization. In order to accelerate the optimization, a priori knowledge is added to the method in the form of parameter ranges.

Comparing the proposed new algorithm to the relevant methods in the field of sensor calibration, it can be stated that there are two main differences. The first one is that this method works with real measurement data, while previous methods work mainly on simulated data. The second one is that this new algorithm is able to calibrate all the three sensors in parallel, while in the previous works only one or maximum two sensors were calibrated.

\section{CALIBRATION AND RESULTS}

For the process of parameter optimization, the evolutionary algorithm was used, because it is well known that it has powerful global optimizing capabilities. The chosen parameters were optimized to give the smallest average error to the expected output. Different fitness functions were constructed depending on the number of parameters which had to be optimized.

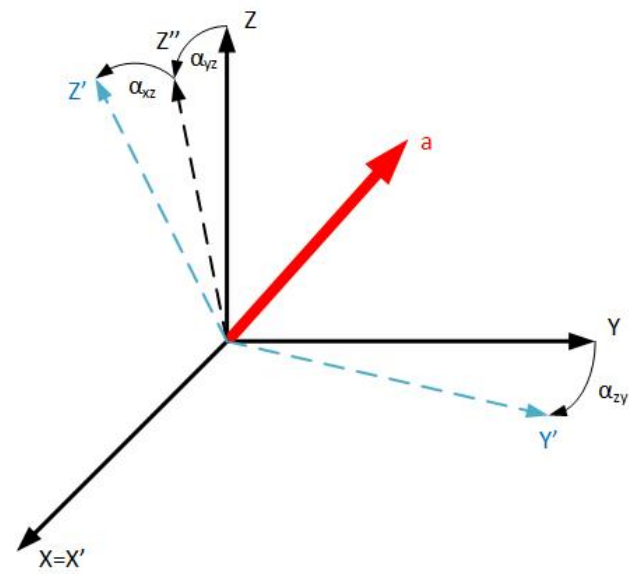

Figure 2. Used model for misalignment angles

\section{A. Accelerometer}

The accelerometers on the three different IRIS motes have been calibrated using different combinations of parameters which should be optimized to find the effect of different error types on the overall error. The axis model used for calculation of misalignment angles can be seen on Fig.2. The functions for magnitude calculation using bias values, scale factors and misalignment angles are showed in (1-5).

$$
\begin{gathered}
X=\left(X^{\prime}+o(1)\right) * s(1) \\
Y=\left(Y^{\prime} * \cos \left(\alpha_{Z Y}\right)+o(2)\right) * s(2) \\
Z^{\prime \prime}=Z^{\prime} * \cos \left(\alpha_{X Z}\right) \\
Z=\left(Z^{\prime \prime} * \cos \left(\alpha_{Y Z}\right)+o(3)\right) * s(3) \\
1=\sqrt{X^{2}+Y^{2}+Z^{2}}
\end{gathered}
$$

where $X^{\prime}, Y^{\prime}$ and $Z^{\prime}$ are the measured values, $X, Y$ and $Z$ are the calibrated values, $o(i)$-s are the offsets, $s(i)$-s are the scale factors, and $\alpha_{i}$-s are the non-orthogonality angles.

The average of the squared errors have been used as the fitness function, where the error is the difference between the calculated outputs with current parameters and the goal value (in this case the goal value is 1 ).

Table 1 shows the used parameters, the errors before and after calibration, and the highest and lowest outputs before and after the calibration of each sensor. The used parameters are dedicated with $X$ in the adequate field of Table 1.

The results show that using only scale factors, or scale factors with misalignment angles have no effect on the calibrated values. The lowest errors have been calculated using all three parameters, but the results show that offsets have the biggest impact on performance. It can be also seen in Table 1 that the misalignment of the axes is very small. According to this, the effect of these parameters is minimal.

The acceleration magnitude levels of one sensor board before and after calibration can be seen on Fig. 3. On Fig. 3 it can be noticed, that from the error levels of $\pm 0.1 \mathrm{~g}$ systematic errors have been removed, and random errors remained in levels between $\pm 25 \mathrm{mg}$. 
TABLE I.

ACCELEROMETER CALIBRATION RESULTS

\begin{tabular}{|c|c|c|c|c|c|c|c|c|c|}
\hline Sensor & $\begin{array}{l}\text { Error } \\
\text { before }\end{array}$ & $\begin{array}{l}\text { Error } \\
\text { after }\end{array}$ & $\begin{array}{c}\text { Lowest output } \\
\text { before }\end{array}$ & $\begin{array}{c}\begin{array}{c}\text { Highest output } \\
\text { before }\end{array} \\
\end{array}$ & $\begin{array}{c}\text { Lowest output } \\
\text { after }\end{array}$ & $\begin{array}{c}\text { Highest output } \\
\text { after }\end{array}$ & Offsets & $\begin{array}{c}\text { Scale } \\
\text { factors }\end{array}$ & $\begin{array}{l}\text { Misalignment } \\
\text { angles }\end{array}$ \\
\hline 1 & 0.001156 & 0.000033 & 0.939532 & 1.075407 & 0.977200 & 1.023137 & $\mathrm{X}$ & & \\
\hline 2 & 0.003806 & 0.000240 & 0.858629 & 1.088883 & 0.945720 & 1.012244 & $\mathrm{X}$ & & \\
\hline 3 & 0.003849 & 0.000110 & 0.877029 & 1.115907 & 0.965846 & 1.021161 & $\mathrm{X}$ & & \\
\hline 1 & 0.001156 & 0.001069 & 0.939532 & 1.075407 & 0.931348 & 1.066069 & & $\mathrm{X}$ & \\
\hline 2 & 0.003806 & 0.002762 & 0.858629 & 1.088883 & 0.901734 & 1.149764 & & $\mathrm{X}$ & \\
\hline 3 & 0.003849 & 0.003127 & 0.877029 & 1.115907 & 0.914001 & 1.166538 & & $\mathrm{X}$ & \\
\hline 1 & 0.001156 & 0.000028 & 0.939532 & 1.075407 & 0.978803 & 1.026313 & $\mathrm{X}$ & $\mathrm{X}$ & \\
\hline 2 & 0.003806 & 0.000034 & 0.858629 & 1.088883 & 0.969710 & 1.024550 & $\mathrm{X}$ & $\mathrm{X}$ & \\
\hline 3 & 0.003849 & 0.000033 & 0.877029 & 1.115907 & 0.974243 & 1.025473 & $\mathrm{X}$ & $\mathrm{X}$ & \\
\hline 1 & 0.001156 & 0.000029 & 0.939532 & 1.075407 & 0.977023 & 1.022959 & $X$ & & $\mathrm{X}$ \\
\hline 2 & 0.003806 & 0.000240 & 0.858629 & 1.088883 & 0.945758 & 1.012210 & $X$ & & $\mathrm{X}$ \\
\hline 3 & 0.003849 & 0.000110 & 0.877029 & 1.115907 & 0.965790 & 1.021219 & $X$ & & $X$ \\
\hline 1 & 0.001156 & 0.001069 & 0.939532 & 1.075407 & 0.931360 & 1.066083 & & $\mathrm{X}$ & $\mathrm{X}$ \\
\hline 2 & 0.003806 & 0.002762 & 0.858629 & 1.088883 & 0.901718 & 1.149742 & & $\mathrm{X}$ & $\mathrm{X}$ \\
\hline 3 & 0.003849 & 0.003127 & 0.877029 & 1.115907 & 0.913998 & 1.166532 & & $\mathrm{X}$ & $\mathrm{X}$ \\
\hline 1 & 0.001156 & 0.000028 & 0.939532 & 1.075407 & 0.979019 & 1.026275 & $\mathrm{X}$ & $\mathrm{X}$ & $\mathrm{X}$ \\
\hline 2 & 0.003806 & 0.000034 & 0.858629 & 1.088883 & 0.969586 & 1.024417 & $\mathrm{X}$ & $X$ & $\mathrm{X}$ \\
\hline 3 & 0.003849 & 0.000033 & 0.877029 & 1.115907 & 0.974416 & 1.025648 & $\mathrm{X}$ & $\mathrm{X}$ & $\mathrm{X}$ \\
\hline
\end{tabular}

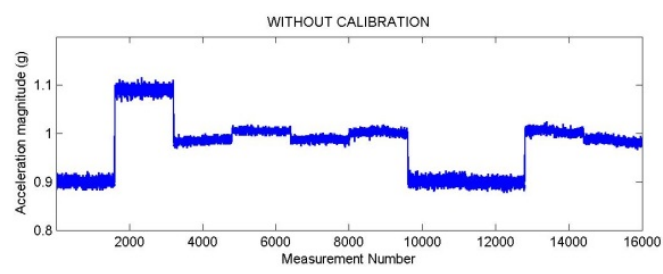

CALIBRATED

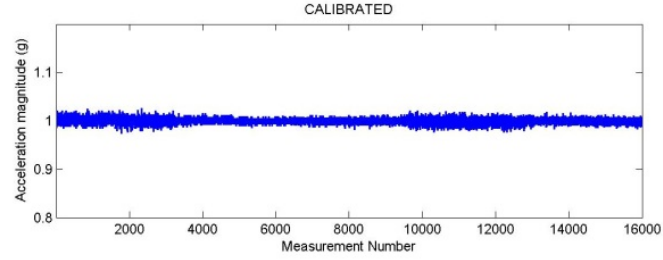

Figure 3. Acceleration magnitude values before and after calibration

\section{B. Gyroscope}

The calibration of gyroscopes has been done only for bias value calculation. The measurement values have been recorded when the sensors were not in motion, so the angular velocity at all three axes should be zero. The fitness function calculates the average squared error from the sum of errors at each axis. The squared error has been calculated with (6).

$$
E=\left(\left|X^{\prime}-b(1)\right|+\left|Y^{\prime}-b(2)\right|+\left|Z^{\prime}-b(3)\right|\right)^{2}
$$

where $E$ is the error, $X^{\prime}, Y^{\prime}$ and $Z^{\prime}$ are the measured values, and $b(i)$-s are the biases.

The results of gyroscope calibration can be seen in Table 2. In addition to the error values before and after calibration, the highest and lowest output values per axis before and after calibration are also presented.

The errors have been reduced significantly, in the case of some axis the bias error was even around $10 \mathrm{deg} / \mathrm{s}$. In the case of all three sensors on the different IRIS motes around $\pm 5 \mathrm{deg} / \mathrm{s}$ noise level still remained at each axis.

\section{Magnetometer}

The magnetometer has been calibrated similarly to the accelerometer, but the expected magnitude was not
TABLE II

GYROSCOPE CALIBRATION RESULTS

\begin{tabular}{|c|c|c|c|}
\hline Sensor & $\mathbf{1}$ & $\mathbf{2}$ & $\mathbf{3}$ \\
\hline Error before & 373.305530 & 312.748027 & 769.303784 \\
\hline Error after & 12.838962 & 11.976923 & 6.251345 \\
\hline $\mathbf{X}-$ lowest value before & -18.1565 & 8.487 & 5.7043 \\
\hline $\mathbf{X}-$ highest value before & -8.1391 & 16.2783 & 14.1913 \\
\hline $\mathbf{X}-$ lowest value after & -5.7741 & -3.5476 & -4.5919 \\
\hline $\mathbf{X}-$ highest value after & 4.2433 & 4.2437 & 3.8951 \\
\hline $\mathbf{Y}-$ lowest value before & -5.2174 & -8.7652 & -19.4783 \\
\hline $\mathbf{Y}-$ highest value before & 9.3217 & 1.4609 & -10.2261 \\
\hline Y - lowest value after & -8.1125 & 6.122 & -5.0782 \\
\hline $\mathbf{Y}-$ highest value after & 6.4266 & 4.1042 & 4.174 \\
\hline $\mathbf{Z}-$ lowest value before & -8.7652 & -2.087 & -6.2609 \\
\hline $\mathbf{Z}-$ highest value before & 0.9739 & 8.0696 & 0.0696 \\
\hline $\mathbf{Z}-$ lowest value after & -4.9391 & -4.8697 & -3.2696 \\
\hline $\mathbf{Z}-$ highest value after & 4.8 & 5.2869 & -2.9217 \\
\hline
\end{tabular}

known, so it was added as another parameter to the fitness function.

The calibration has been done with two types of data: measurements made at fix orientations, and measurements recorded when rotating the sensor.

Due to the big shifts from the coordinate system's origin, and the deformations of the sphere which can be recognized on the three-dimensional plots, it was pointless to make optimizations when only offsets or scale factors are used. Biases and scale factors have been optimized with and without non-orthogonality angles.

Table 3 shows the optimized magnitudes, error levels, smallest and highest magnitude outputs with applying only offsets, and with all three parameter types used. $O$ in the used data set column in Table 3 means that the optimization has been done with data recorded in fix orientations, while $R$ means that the measurements were recorded during rotation of the sensor have been used. $X$ in the misalignment angles column in Table 3 indicates that non-orthogonality angles have been also optimized.

The magnitude outputs of one sensor before calibration and after calibration using all three parameters can be seen on Fig. 4. 
TABLE III

MAGNETOMETER CALIBRATION RESULTS

\begin{tabular}{|c|c|c|c|c|c|c|c|c|c|}
\hline Sensor & $\begin{array}{c}\text { Used } \\
\text { data } \\
\text { set } \\
\end{array}$ & $\begin{array}{l}\text { Optimized } \\
\text { magnitude }\end{array}$ & $\begin{array}{c}\text { Error with } \\
\text { offsets }\end{array}$ & $\begin{array}{c}\text { Error with all } \\
\text { parameters }\end{array}$ & $\begin{array}{c}\text { Lowest } \\
\text { output with } \\
\text { offsets } \\
\end{array}$ & $\begin{array}{c}\text { Highest output } \\
\text { with offsets }\end{array}$ & \begin{tabular}{|c|} 
Lowest output \\
with all \\
parameters \\
\end{tabular} & $\begin{array}{c}\text { Highest output } \\
\text { with all } \\
\text { parameters } \\
\end{array}$ & $\begin{array}{c}\text { Misalignment } \\
\text { angles }\end{array}$ \\
\hline 1 & $\mathrm{R}$ & 0.434058 & 0.000896 & 0.000183 & 0.379845 & 0.507250 & 0.397041 & 0.472039 & \\
\hline 2 & $\mathrm{R}$ & 0.471452 & 0.001508 & 0.000311 & 0.388275 & 0.562161 & 0.419891 & 0.513897 & \\
\hline 3 & $\mathrm{R}$ & 0.499004 & 0.000663 & 0.000217 & 0.448080 & 0.561891 & 0.462450 & 0.529454 & \\
\hline 1 & $\mathrm{O}$ & 0.539963 & 0.001401 & 0.000257 & 0.470094 & 0.626799 & 0.505491 & 0.578175 & \\
\hline 2 & $\mathrm{O}$ & 0.458486 & 0.001889 & 0.000243 & 0.384346 & 0.547930 & 0.420813 & 0.495475 & \\
\hline 3 & $\mathrm{O}$ & 0.488890 & 0.000674 & 0.000180 & 0.443340 & 0.555447 & 0.456065 & 0.519531 & \\
\hline 1 & $\mathrm{R}$ & 0.436494 & 0.000818 & 0.000184 & 0.379329 & 0.507534 & 0.397341 & 0.473531 & $\mathrm{X}$ \\
\hline 2 & $\mathrm{R}$ & 0.470633 & 0.001546 & 0.000301 & 0.387697 & 0.563049 & 0.419029 & 0.512368 & $\mathrm{X}$ \\
\hline 3 & $\mathrm{R}$ & 0.485687 & 0.000866 & 0.000206 & 0.448051 & 0.561108 & 0.449906 & 0.516376 & $\mathrm{X}$ \\
\hline 1 & $\mathrm{O}$ & 0.539499 & 0.001414 & 0.000255 & 0.470114 & 0.626574 & 0.504527 & 0.576421 & $\mathrm{X}$ \\
\hline 2 & $\mathrm{O}$ & 0.456709 & 0.001948 & 0.000233 & 0.384885 & 0.547896 & 0.421367 & 0.493234 & $\mathrm{X}$ \\
\hline 3 & $\mathrm{O}$ & 0.482189 & 0.000698 & 0.000175 & 0.443712 & 0.555554 & 0.449264 & 0.512025 & $\mathrm{X}$ \\
\hline
\end{tabular}

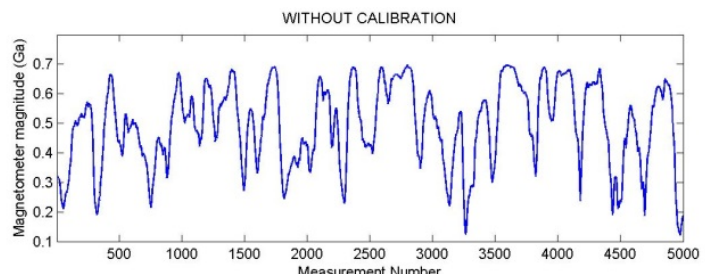

CALIBRATED

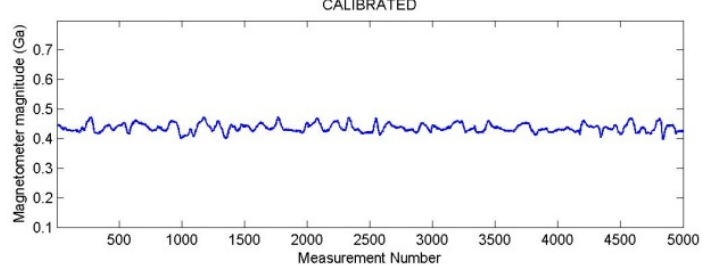

Figure 4. Magnetometer magnitude values before and after calibration

Fig. 5 and Fig. 6 show the positions in 3D and in the three planes before and after calibration at one of the sensor's measurements with the data set of the rotated sensor. The results show that even with using all three types of parameters, neither of the data sets can be regularly calibrated. Error levels are lot smaller than when no calibration is done, but they are still too high. Scale factors have bigger effect compared to the accelerometers, but the misalignment angles are very small, and thus, have small effect on the error.

\section{CONCLUSION}

In this paper a new, evolutionary algorithm based, 9DOF sensor board calibration process has been discussed. The used measurement data have been recorded with three sensor boards attached to IRIS WSN motes, since these small, portable and light weight units can easily be used in later stages of the medical emergency detection project.

Although non-linearity and thermal calibration were not investigated yet, enhancements were reached in the compensation of systematic errors of the applied sensors.

The accelerometer calibration results show that the systematic error can be completely removed, and the errors can be reduced to only random errors. The error levels with different parameter combinations indicate that

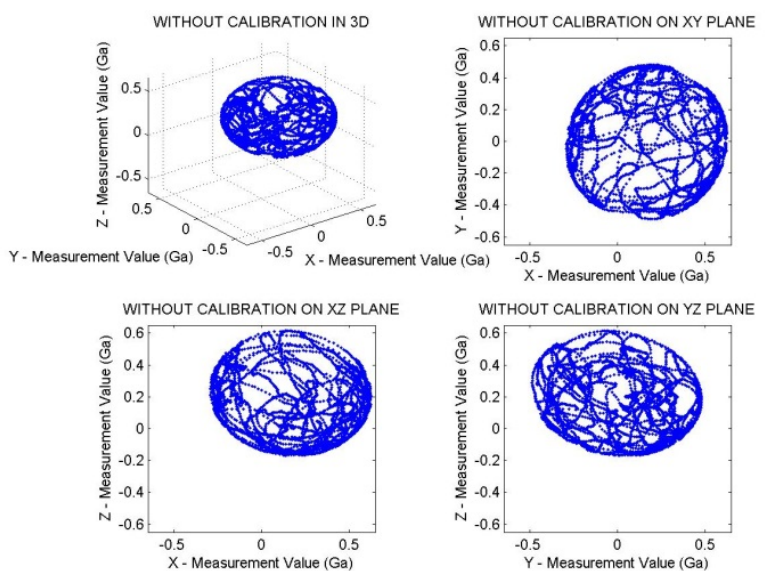

Figure 5. Raw measurement values in $3 \mathrm{D}$ and in the three planes with the data set where the sensor was rotated

the misalignments between the axes are very small, and almost do not have any effect on the output values.

The gyroscopes have been only calibrated to filter bias errors, which have the biggest effect on the measurements, and which in some cases are very high. After removing this systematic error, only noise levels remained. The method should be expanded with some kind of scale factor and misalignment calibration which does not need special equipment.

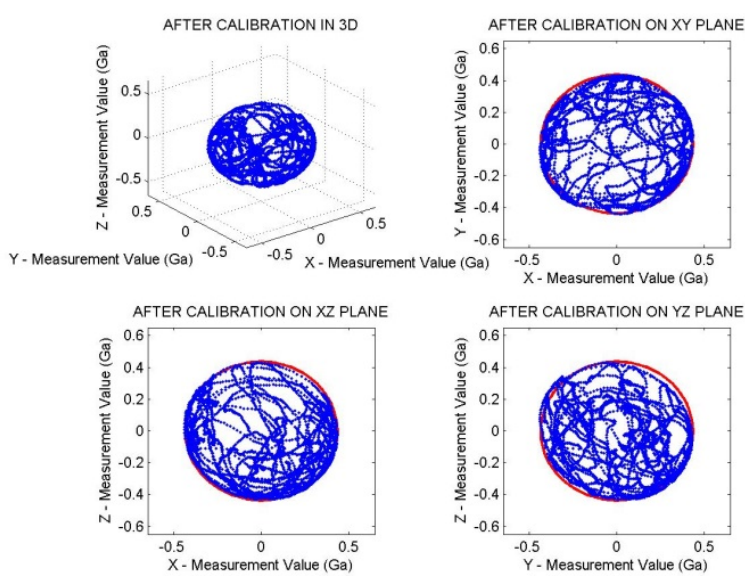

Figure 6. Calibrated values in 3D and in the three planes with the data set where the sensor was rotated 
Magnetometer calibration has been done with two types of data. First, measurements were recorded at fix orientations, and then data were collected when the sensors were rotated. The results show that the error levels can be significantly reduced, but could not be totally removed in the case of neither of the data sets. The scale factors have bigger effect compared to the accelerometers, but the misalignment angles are similarly very small. An improved calibration method is needed to filter out remaining error sources.

The proposed evolutionary algorithm based sensor calibration method was also compared to the relevant methods in the field of sensor calibration.

\section{ACKNOWLEDGMENT}

The publication is supported by the European Union and co-funded by the European Social Fund. Project title: "Telemedicine-focused research activities on the field of Mathematics, Informatics and Medical sciences" Project number: TÁMOP-4.2.2.A-11/1/KONV-2012-0073.

\section{REFERENCES}

[1] I. Frosio, F. Pedersini, and N. A. Borghese, "Autocalibration of MEMS Accelerometers", Advanced Mechatronics and MEMS Devices, Mycrosystems Vol. 23, 2013, pp. 53-88.

[2] M. Gietzelt, K.-H. Wolf, M. Marschollek, and R. Haux, "Performance comparison of accelerometer calibration algorithms based on 3D-ellipsoid fitting methods", Computer Methods and Programs in Biomedicine, Vol. 111, Elsevier, 2013, pp. 62-71.

[3] P. Batista, C. Silvestre, P. Oliveira, and B. Cardeira, "Accelerometer Calibration and Dynamic Bias and Gravity Estimation: Analysis, Design, and Experimental Evaluation", IEEE Transactions on Control Systems Technology, Vol. 19, 2011, pp.1128-1137.
[4] M. Glueck, D. Oshinubi, and Y. Manoli, "Automatic real-time offset calibration of gyroscopes", IEEE Sensor Applications Symposium, 2013, pp. 214-218.

[5] D. Jurman, M. Jankovec, R. Kamnik, and M. Topic, "Calibration and data fusion solution for the miniature attitude and heading reference system", Sensors and Actuators A: Physical, Vol. 138, Elsevier, 2007, pp. 411-420.

[6] I. Skog, and P. Handel, "Calibration of a MEMS inertial measurement unit", XVII IMEKO World Congress, Rio de Janeiro, Brazil, 2006.

[7] M.-S. Kim, S.-B. Yu, and K.-S. Lee, "Development of a HighPrecision Calibration Method for Inertial Measurement Unit", International Journal of Precision Engineering and Manufacturing, Vol. 15, 2014, pp. 567-575.

[8] J. L. Crassidis, K.-L. Lai, and R. R. Harman, "Real-time attitudeindependent three-axis magnetometer calibration", Journal of Guidance, Control and Dynamics, Vol. 28, 2005, pp. 115-120.

[9] J.F. Vasconcelos, G. Elkaim, C. Silvestre, P. Oliveira, and B. Cardeira, "A Geometric Approach to Strapdown Magnetometer Calibration in Sensor Frame", IEEE Transactions on Aerospace and Electronics Systems, Vol. 47, 2011, pp. 1293-1306.

[10] D. Gebre-Egziabher, G. H. Elkaimy, J. D. Powellz, and B. W. Parkinson, "A non-linear, two-step estimation algorithm for calibrating solid-state strapdown magnetometers", $8^{\text {th }}$ International St. Petersburg conference on navigation systems, St. Petersburg, Russia, 2001.

[11] H. Pang, et al., "Calibration of three-axis magnetometers with differential evolution algorithm", Journal of Magnetism and Magnetic Materials, Vol. 346, 2013, pp. 5-10.

[12] S. Luo, et al., "Calibration strategy and generality test of threeaxis magnetometers", Measurement, Vol. 46, 2013, 2013, pp. 3918-3923.

[13] V. Renaudin, M. H. Azfal, and G. Lachapelle, "Complete Triaxis Magnetometer Calibration in the Magnetic Domain", Journal Of Sensors, Vol. 2010, 2010, p.10.

[14] F.-X. Wu, B. Hua, and G.-H.Kang, "Error Calibration of Tri-axial Magnetometer Based on Particle Swarm Optimization Algorithm", China Satellite Navigation Conference (CSNC) 2014 Proceedings: Volume III, Lecture Notes in Electrical Engineering, Vol. 305, 2014, pp 577-584. 